\title{
Pulmonary endarterectomy and balloon pulmonary angioplasty in chronic thromboembolic pulmonary hypertension: The Singapore experience
}

\section{Dear Editor,}

Chronic thromboembolic pulmonary hypertension (CTEPH) is a pulmonary hypertension caused by mechanical obstruction of the pulmonary arteries by organised thrombi and microvascular arteriopathy. It occurs as a complication of pulmonary embolism, and if left untreated, results in right heart failure and death. ${ }^{1}$ The gold standard for treatment is pulmonary endarterectomy (PEA) which removes obstructive thromboemboli surgically. For inoperable cases, balloon pulmonary angioplasty (BPA) is an alternative that uses balloon dilatation to open up the arteries. Medical therapy is reserved for cases that are not suitable for PEA, BPA, or residual pulmonary hypertension occurring post-procedures.

We established the first CTEPH multidisciplinary team in Singapore and conducted a retrospective review of all consecutive patients who underwent PEA or BPA for CTEPH at our tertiary cardiac centre from June 2015 to January 2019. All patients were evaluated by the team after diagnosis. ${ }^{2}$ Efficacy outcomes studied included change in New York Heart Association (NYHA) functional class and pulmonary haemodynamics. Safety outcomes included peri-procedural complications and mortality.

Clinical characteristics. There were $25 \mathrm{CTEPH}$ cases, of which 16 underwent PEA and 11 underwent BPA (2 had residual pulmonary hypertension postPEA). The median age was 55 years (interquartile range [IQR] 26-77), with the majority being women (68\%) (Table 1). All patients underwent baseline cardiac catheterisation and $88 \%$ of them had invasive pulmonary angiography. Baseline mean pulmonary artery pressure (PAP) was 44.3mmHg (standard deviation [SD] 9.4) and pulmonary vascular resistance (PVR) was $9.3 \mathrm{Wu}$

Table 1. Baseline clinical characteristics of study population

\begin{tabular}{|c|c|c|c|}
\hline & $\begin{array}{l}\text { Total } \\
\mathbf{N}=\mathbf{2 5}\end{array}$ & $\begin{array}{l}\text { PEA } \\
n=16\end{array}$ & $\begin{array}{l}\text { BPA } \\
n=11^{\text {a }}\end{array}$ \\
\hline Age, mean (SD), years & $55.7(12.6)$ & $54.8(9.9)$ & $57.0(16.1)$ \\
\hline Women, no. (\%) & $17(68.0)$ & $10(62.5)$ & $8(72.7)$ \\
\hline BMI (SD) & $25.3(3.8)$ & $26.4(2.9)$ & $24.8(5.2)$ \\
\hline Blood group (Non-O), no. (\%) & $15(60.0)$ & $13(81.3)$ & $4(36.4)$ \\
\hline History of acute PE, no. (\%) & $20(80.0)$ & $12(75.0)$ & $10(90.9)$ \\
\hline History of DVT, no. (\%) & $10(40.0)$ & $8(50.0)$ & $3(27.3)$ \\
\hline History of IVC insertion, no. (\%) & $3(12.0)$ & $2(12.5)$ & $2(18.2)$ \\
\hline \multicolumn{4}{|l|}{ Comorbidities } \\
\hline CAD/IHD, no. (\%) & $5(20.0)$ & $4(25.0)$ & $2(18.2)$ \\
\hline Hypertension, no. (\%) & $10(40.0)$ & $6(37.5)$ & $5(45.5)$ \\
\hline Dyslipidaemia, no. (\%) & $9(36.0)$ & $6(37.5)$ & $5(45.5)$ \\
\hline Diabetes mellitus, no. (\%) & $6(24.0)$ & $4(25.0)$ & $3(27.3)$ \\
\hline $\begin{array}{l}\text { Thrombophilic disorder, no. (\%) } \\
\text { Protein C or protein S deficiency, no. (\%) } \\
\text { Antiphospholipid syndrome, no. (\%) }\end{array}$ & $\begin{array}{l}5(20.0) \\
2(40.0) \\
3(60.0)\end{array}$ & $\begin{array}{l}4(25.0) \\
1(25.0) \\
3(75.0)\end{array}$ & $\begin{array}{l}1(9.1) \\
1(100.0)\end{array}$ \\
\hline Cancer, no. $(\%)$ & $2(8.0)$ & $2(12.5)$ & $0(0)$ \\
\hline Smoking, no. (\%) & $6(24.0)$ & $6(37.5)$ & $1(9.1)$ \\
\hline
\end{tabular}

BPA: balloon pulmonary angioplasty; CAD/IHD: coronary artery disease/ischaemic heart disease; DVT: deep vein thrombosis; IVC: inferior vena cava; PE: pulmonary embolisation; PEA: pulmonary endarterectomy; SD: standard deviation

a 2 patients who had prior PEA underwent BPA for residual disease 
(SD 5.3). There were 3 patients who had coronary artery revascularisation.

The commonest presentations in patients were: breathlessness on exertion $15(60 \%)$; fatigue $7(28 \%)$; and peripheral oedema $2(8 \%)$. Baseline NYHA classes were II or III, with a mean 6-minute walk distance (6MWD) of $396 \mathrm{~m}$ (SD 156). Patients had a median of 105 days (IQR 29-180) of symptoms before presentation. Prior to intervention, $3(12 \%)$ required home oxygen therapy; $14(56 \%)$ were on pulmonary vasodilators; 19 (76\%) on warfarin; $5(20 \%)$ on direct oral anticoagulation; and $1(4 \%)$ on low molecular weight heparin.

Pulmonary endarterectomy. A total of 16 PEAs were performed. Phase 1 of the programme (pre-proctorship) was from June 2015 to June 2016, during which 4 PEAs were performed with $2(50 \%)$ in-hospital deaths. Phase 2 started after a Health Manpower Development Programme attachment to Papworth Hospital, UK in June 2016, where 10 proctored surgeries were performed. In Phase 3 (post-proctorship), 2 PEAs were done by local surgeons. No in-hospital mortality occurred among the 12 cases in phase 2 and 3. One patient required temporary veno-venous extracorporeal membrane oxygenation (ECMO) support for reperfusion lung injury. Seven cases of post-operative subdural haemorrhage were noted (1 required Burr hole drainage, while the rest were conservatively treated). In the surviving 14 patients, at 36 months postPEA, there was a significant reduction in PVR from $11.6 \mathrm{Wu}$ (SD 5.2) to 4.2Wu (SD 3.7) $(P=0.008)$. Mean PAP reduced from $48.6 \mathrm{mmHg}$ (SD 5.5) to $30.4 \mathrm{mmHg}$ (SD 16.4) $(P<0.001)$, and $\mathrm{N}$-terminal prohormone B-type natriuretic peptide from $1,441 \mathrm{pg} /$ $\mathrm{mL}(\mathrm{SD} 1,847)$ to $201 \mathrm{pg} / \mathrm{mL}$ (SD 153) $(P=0.010)$. There was improvement in cardiac output from $3.1 \mathrm{~L} / \mathrm{min}(\mathrm{SD} 1.1)$ to $4.2 \mathrm{~L} / \mathrm{min}$ (SD 0.4) $(P=0.004)$, 6MWD from $357 \mathrm{~m}$ (SD 104) to $417 \mathrm{~m}$ (SD 87), and NYHA class to I and II $(P=0.003)$.

At 3-6 months post-PEA, 11 (69\%) patients were able to stop pulmonary vasodilators. Three (19\%) had persistent pre-capillary pulmonary arterial hypertension. Two of them had residual disease deemed suitable for BPA (perfusion defects on dual-energy computed tomography pulmonary angiography corresponding to anatomical obstruction). The remaining patient was likely small vessel CTEPH and was treated with medication.

Balloon pulmonary angioplasty. A total of $49 \mathrm{BPA}$ sessions were performed for 11 patients, averaging 4.5 sessions per patient, and 7 (SD 2) segments per session.
Five patients $(45.5 \%)$ completed BPA, 5 (45.5\%) had ongoing sessions, while $1(9 \%)$ declined further sessions. Three patients (27\%) had BPA for persistent pulmonary hypertension after PEA. Technical (lesion) success rate, defined as lesion crossing and dilatation without flow-limiting major dissection, was over $90 \%$. There were no major complications such as inhospital death, stroke, and severe lung injury requiring mechanical ventilation or ECMO support. Guidewirerelated perforation of segmental pulmonary arteries, manifesting as recurrent cough and haemoptysis, occurred in 4 sessions (8\%). All were successfully managed with gel foam embolisation. In the 8 patients who had completed at least 3 sessions and at least 6 months of post-BPA follow-up, there were reduction of mean PAP from $40.9 \mathrm{mmHg}$ (SD 9.6) to $28.5 \mathrm{mmHg}$ (SD 7.7), and PVR from $6.5 \mathrm{Wu}$ (SD 1.5) to $3.8 \mathrm{Wu}$ (SD 1.7). There was modest improvement in cardiac output from $4.3 \mathrm{~L} / \mathrm{min}$ (SD 0.7) to $4.6 \mathrm{~L} / \mathrm{min}$ (SD 0.7), and NYHA functional class by at least 1 grade. Of the remaining 3 patients, 2 are still undergoing treatment and reporting symptomatic improvement; 1 had multiple respiratory comorbidities and residual pulmonary hypertension after previous PEA, and did not have symptomatic or haemodynamic improvement after 2 BPA sessions (Table 2).

Interventional options. To the best of our knowledge, this is the first multidisciplinary therapeutic management of CTEPH in Southeast Asia. The pros and cons of different imaging modalities and our institution's preference (i.e. dual-energy computed tomography pulmonary angiography) have been previously discussed. ${ }^{3}$ Although our study number was small, we report satisfactory survival rates and functional outcomes.

PEA is a sophisticated surgery with a steep learning curve. It requires cardiopulmonary bypass and deep hypothermic circulatory arrest to enable a complete endarterectomy. The surgery involves dissection into subsegmental branches of pulmonary arteries, a technically demanding procedure that requires specialist training. Successful surgery also depends on specific intensive care peri- and post-operatively. ${ }^{4}$ In-hospital mortality rates range from $\leq 3.5-7.4 \%$ or higher depending on centre experiences. ${ }^{5}$ In our experience, with proctorship and greater team experience, PEA outcomes improved significantly. The unexpectedly high initial incidence of subdural haemorrhage prompted a modification of the protocol, to be less aggressive in achieving negative fluid balance and to initiate anticoagulation only after computed tomography of brain suggests no evidence of bleeding post- 


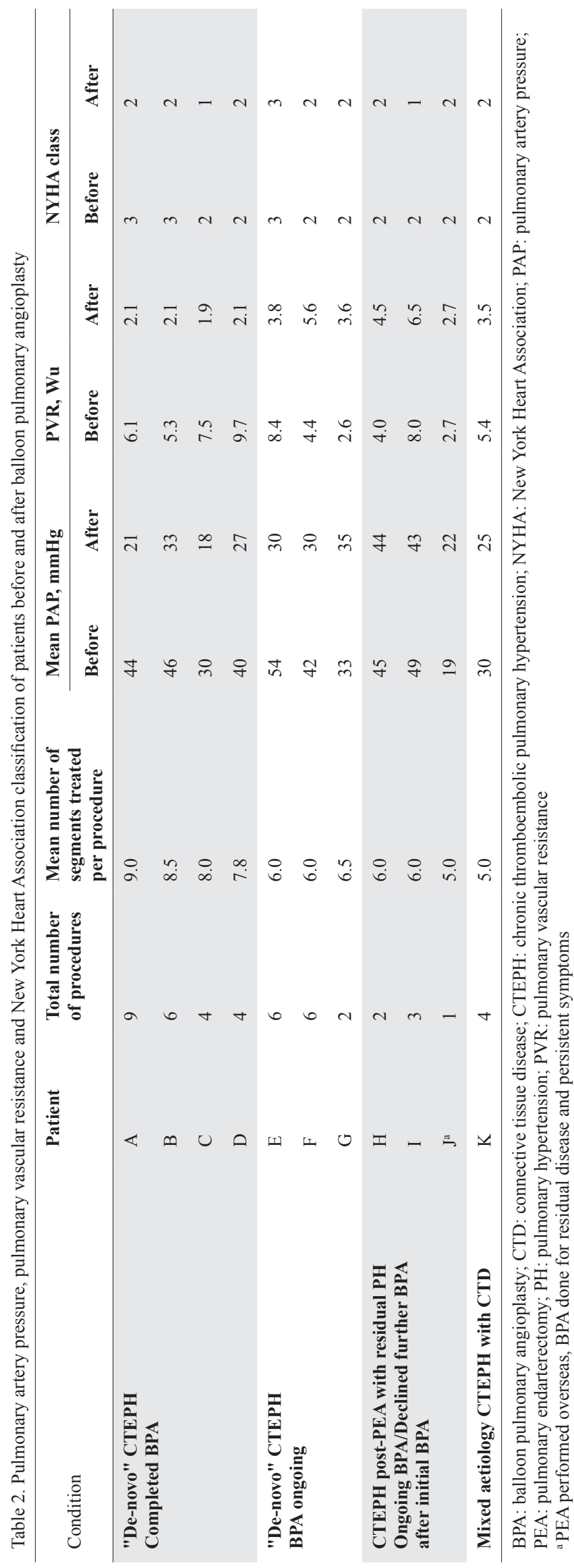

operatively. ${ }^{6}$ This has been shown to better suit the Singapore population.

BPA is reserved for CTEPH patients with technically inoperable disease or with unfavourable risk-to-benefit ratio for surgery. ${ }^{2}$ It is not a first-line treatment because it does not physically remove the organised thrombi. Instead, it improves haemodynamics by compressing obstruction to the side of vessel walls with sequential balloon dilatation. As such, BPA is usually reserved for CTEPH involving smaller vessels. In appropriately selected cases and with experience, BPA has been shown to significantly improve patient symptoms, exercise tolerance and pulmonary haemodynamics, without major procedural complications. ${ }^{7}$

Regional experience. Due to the complexity of PEA, much effort and training is needed in Asia Pacific before it becomes widely adopted. In Japan, PEA was carried out in 73 patients in 2017 with a 30-day mortality of $4.1 \%$ and in-hospital mortality of 9.6\%. ${ }^{8}$ In China, Australia, South Korea, Taiwan and India, single-centre studies with smaller numbers have been reported from 1994-2010, with 30-day mortalities ranging from $0-12.19 \%{ }^{9-13}$ For BPA, Japan first reported 308 CTEPH patients who underwent 1,408 BPA procedures between 2004 and 2013 at 7 institutions. The overall 3-year survival rate was 94.5\%. ${ }^{7}$ Several other Asian Pacific countries have also published single-centre experiences in small numbers ( $<50$ total cases), with improvements in haemodynamics, symptoms and exercise capacity, as well as generally low rates of major complications and mortality. ${ }^{14,15}$ The management of CTEPH patients requires a multidisciplinary team for consistent and beneficial outcomes. We have shown PEA and BPA to be safe and efficacious in suitable patients. With experience, BPA is poised to play a greater role in the management of CTEPH in the region.

\section{REFERENCES}

1. Lang I. Chronic thromboembolic pulmonary hypertension: a distinct disease entity. Eur Respir Rev 2015;24:246-52.

2. Galiè N, Humbert M, Vachiery JL, et al. 2015 ESC/ERS Guidelines for the diagnosis and treatment of pulmonary hypertension: the joint task force for the diagnosis and treatment of pulmonary hypertension of the European Society of Cardiology (ESC) and the European Respiratory Society (ERS). Eur Heart J 2016;37:67-119.

3. Ruan W, Yap JJ, Quah KK, et al. Clinical updates on the diagnosis and management of chronic thromboembolic pulmonary hypertension. Ann Acad Med Singap 2020;49:320-30.

4. Jenkins D. Pulmonary endarterectomy: the potentially curative treatment for patients with chronic thromboembolic pulmonary hypertension. Eur Respir Rev 2015;24:263-71. 
5. Madani MM, Auger WR, Pretorius V, et al. Pulmonary endarterectomy: recent changes in a single institution's experience of more than 2,700 patients. Ann Thorac Surg 2012;94:97-103.

6. Yap J, Ruan W, Chia A, et al. Is subdural hemorrhage after pulmonary endarterectomy underrecognized? J Thorac Cardiovasc Surg 2018;156:2039-42.

7. Ogawa A, Satoh T, Fukuda T, et al. Balloon pulmonary angioplasty for chronic thromboembolic pulmonary hypertension: results of a multicenter registry. Circ Cardiovasc Qual Outcomes 2017; 10:e04029.

8. Committee for Scientific Affairs, The Japanese Association for Thoracic Surgery, et al. Thoracic and cardiovascular surgeries in Japan during 2017: Annual report by the Japanese Association for Thoracic Surgery. Gen Thorac Cardiovasc Surg 2020;68:414-49.

9. Gan HL, Zhang JQ, Bo P, et al. The actuarial survival analysis of the surgical and non-surgical therapy regimen for chronic thromboembolic pulmonary hypertension. J Thromb Thrombolysis 2010;29:25-31.

10. Narayana Iyengar RM, Hegde D, Chattuparambil B, et al. Postoperative management of pulmonary endarterectomy and outcome. Ann Card Anaesth 2010;13:22-7.

11. Luo WC, Huang SC, Lin YH, et al. Pulmonary endarterectomy for chronic thromboembolic pulmonary hypertension-A single-center experience in Taiwan. J Formos Med Assoc 2015;114:1197-1203.

12. Park SY, Lee SM, Shin JW, et al. Epidemiology of chronic thromboembolic pulmonary hypertension in Korea: results from the Korean registry. Korean J Intern Med 2016;31:305-12.

13. Maliyasena VA, Hopkins PM, Thomson BM, et al. An Australian tertiary referral center experience of the management of chronic thromboembolic pulmonary hypertension. Pulm Circ 2012; 2:359-64.
14. Kwon W, Yang JH, Park TK, et al. Impact of balloon pulmonary angioplasty on hemodynamics and clinical outcomes in patients with chronic thromboembolic pulmonary hypertension: the initial Korean experience. J Korean Med Sci 2018;33:e24.

15. Chen ZW, Wu CK, Kuo PH, et al. Efficacy and safety of balloon pulmonary angioplasty in patients with inoperable chronic thromboembolic pulmonary hypertension. J Formos Med Assoc 2021;120:947-55.

Wen $\underline{\text { Ruan }},{ }^{* 1} M R C P(U K)$, Jonathan Yap, ${ }^{* 1} M R C P(U K)$,

Kevin Quah, ${ }^{1}{ }_{M R C P}(U K)$, Foong Koon Cheah, ${ }^{2}{ }_{F R C R}$,

Ghee Chee Phua, ${ }^{3}{ }_{M R C P}(U K)$, Duu Wen Sewa, ${ }^{3}{ }_{M R C P}(U K)$,

Aidila Binte Ismail, ${ }^{1}$, Alicia Chia, ${ }^{4}{ }_{F R C S E d}(C T h)$,

David Jenkins, ${ }^{5}{ }_{F R C S}$, Ju Le Tan, ${ }^{1}{ }_{M R C P(U K)}$,

Victor $\underline{\text { Chao }},{ }^{4}{ }_{F R C S}$, Soo Teik Lim, ${ }^{1}{ }_{M R C P}(U K)$

\footnotetext{
${ }^{1}$ Department of Cardiology, National Heart Centre, Singapore

${ }^{2}$ Department of Radiology, Singapore General Hospital, Singapore

${ }^{3}$ Department of Respiratory Medicine and Critical Care, Singapore General Hospital, Singapore

${ }^{4}$ Department of Cardiothoracic Surgery, National Heart Centre, Singapore

${ }^{5}$ Royal Papworth Hospital, United Kingdom
}

Correspondence: Dr Wen Ruan, Department of Cardiology, National Heart Centre, 5 Hospital Drive, Singapore 169609.

Email: ruan.wen@singhealth.com.sg

* Joint first authors 